\title{
A Coordination Framework for Distributed Supply Chains
}

\author{
H. K. Chan \\ Department of Industrial \& Manufacturing Systems \\ Engineering \\ The University of Hong Kong, Hong Kong \\ hkchan@ieee.org
}

\begin{abstract}
Supply chain is a network of cooperating organizations that are involved through upstream and downstream linkages. Such a complex system cannot be modeled simply by mathematical equations without simplification of the problem. Recently, Multi-Agent System (MAS) is proposed as a modeling technique to represent supply chain networks. In fact, many' application problems in MASs that are concerned with finding a consistent combination of agent actions can be formalized as Distributed Constraints Satisfaction Problem (DCSP). The main purpose of this paper is to propose a novel coordination framework by adopting the DCSP philosophy for distributed supply chains, which are modeled by MASsi, subjected to uncertainties. Simulation results indicate that the proposed mechanism outperforms traditional stochastic modeling in solving supply' chain dynamics in terms of total cosi and fill rate of the system.
\end{abstract}

Keywords: Supply Chain Coordination and Optimization, Modeling and Simulation of Supply Chain Management.

\section{Introduction}

Supply chain can be viewed as a network of participating corporations working together to achieve the system goals. Supply chain management is the act of optimizing all activities through the supply chain, so that products and services are supplied in the right quantity, to the right time, and at the optimal cost. In this connection, coordination among supply chain members is of vital importance. Due to the distributed nature of global supply chain, agent technology has been employed to model supply chains. Agent technology provides methods of integrating the entire supply chain as a networked systern of independent echelons, each of which utilizes its own decision-making procedure [1]. Mutli-agent system (MAS), a branch of Distributed Artificial Intelligence, consists of more than one autonomous agent. Coordination, or teamwork, is one of the critical research challenges in a large number of multi-agent applications [2]. In fact, many application problems in MASs that are concerned with finding a consistent combination of agent actions can be

\footnotetext{
* 0-7803-8566-7/04/\$20.00 $\odot 2004$ IEEE.
}

\author{
Felix T. S. Chan \\ Department of Industrial \& Manufacturing \\ Systems Engineering \\ The University of Hong Kong, Hong Kong \\ ftschan@hkucc.hku.hk
}

formalized as Distributed Constraints Satisfaction Problem (DCSP). In this connection, this paper aims to develop a coordination methodology, which is based on DCSP, through quantity flexibility to resolve supply chain dynamics.

The rest of the paper is organized as follows: Section 2 provides a review of related literature. Section 3 formulates the simulation problems, in terms of stochastic model and the proposed coordination mechanism. Section 4 presents and discusses the simulation results. Section 5 is the concluding section.

\section{Literature review}

\subsection{Coordination in supply chains}

Quantity / price discount is a common strategy to provide coordination . channel among supply chain members. For example, Viswanathan and Piplani [3] considered a policy where a vendor offers a discount to buyers as an incentive for them to place orders only at times specified by the vendor. One common weakness of the reported research with such channel coordination is that deterministic demand is assumed, and hence, impact of system dynamics on the proposed model has not been studied. Facing uncertain demand, for example, retailers prefer to place an order late in most case in order to gather enough time to collect more information [4]. However, this leads to insufficient production times and hence production cost would probably be increased.

Coordination can also exist in the form of contracting. Quantity flexibility contract provides flexibility with no explicit penalty for exercise, by adopting constraints as a way to motivate appropriate behavior [5]. This philosophy is in line with solving Constraint Satisfaction Problem (CSP), which will be reviewed in the later sub-section 2.3. By introducing quantity flexibility, the retailer can place an order earlier due to the flexibility that is introduced in the quantity range and the supplier may only need to finish the order with quantity that is within the committed range. In addition, the retailer may request less quantity of goods to be shipped if the actual demand is lower than what is 
expected. This philosophy can provide incentive to both supplier and retailer.

\subsection{Distributed problem solving in supply chains}

Multi-Agent System (MAS) is a typical example of distributed problem solving technique that gains high attention in recent research. Swaminathan et al. [6] presented a multi-agent approach to model supply chain dynamics. A supply chain library of software components has been developed such that customized supply chain models can be built from the library. Sadeh et al. [7] presented an agent-based architecture for dynamic supply chain called MASCOT (Multi-Agent Supply Chain cOordination Tool). MASCOT is a re-configurable, multilevel, agent-based architecture for coordinated supply chain. Agents in MASCOT serve as wrappers for planning and scheduling modules. Above mentioned architectures are focusing on the architectural issues and lacking of higher coordination mechanism. Since agents in a MAS is loosely coupled and are not controlled by a central controller, it is easy to loss distributed functions. Coordination is an effective tool to prevent the system from such problem, i.e. chaotic behavior in agent's terminology.

\subsection{Distributed constraint satisfaction problem}

Constraint Satisfaction Problem (CSP) consists of a set of variables $\mathrm{X}=\left\{x_{l}, \ldots, x_{n}\right\}$ while each variable $x_{i}$ belongs to a finite set $D_{i}$ of possible values (its domain), and a set of constraints restricting the values that the variables can simultaneously assign from the domain. The objective is to find values of the variables that satisfy every constraint. Many problems in operational research, e.g. scheduling, fall within this general framework. Distributed CSP (DCSP) is formally defined as a CSP in which variables and constraints are distributed among multiple automated agents [8]. Various application problems in MAS that are concerned with finding a consistent combination of agent actions can be formalized as DCSP while solving DCSP involves communication between agents by sending message and each agent has some variables and tries to determine their values so that the value assignment must satisfy inter-agent constraints, which are also distributed among agents [9].

In this paper, the philosophy of DCSP is applied to solve inventory management problem in distributed supply chains. In an inventory management problem, the variables of a retailer are the quantity and the time to re-order, which decision is constrained by its inventory position. For supplier, the variables are the quantity to produce and the time to ship (if the supplier has the flexibility), which is constrained by its own capacity and raw material supply. Domain of the variables is simply positive integers. Details will be provided in the next section.

\section{Problem formulation}

The supply chain under study is modeled as the MAS that described in the previous research work that was conducted by Chan and Chan [10]. Since the main objective of this paper is to discuss the coordination mechanism for inventory management through flexible quantity in distributed supply chains, the model and details of operations are not reproduced here. Interest readers can refer to the previous work for details [10]. In summary, independent companies are represented by agents. In fact, the coordination mechanism is relied on inter-agent communication and negotiation to come up with how and when to deliver outstanding orders in supply chain, with the background philosophy of solving CSP. Each agent's action is governed by constraints and the performance of the coordination mechanism is compared against traditional stochastic model in order to verify the usefulness of the proposed mechanism.

\subsection{Stochastic model}

The supply chain under study consists of one retailer and four suppliers. They are independent in the way that suppliers do not have access to the demand information and the retailer does not know the capacity of the suppliers. Each supplier's capacity per period is limited and is uncertain. Sequences of operations are stated as follows:

- Retailer's inventory at period $t\left(I_{t}\right)$ is reduced according to daily demand $\left(d_{t}\right)$, which is normally distributed, plus all incoming orders $\left(I O_{t}\right)$ from supplier $i$ at period $t$. Any unfilled demand is backordered $\left(B O_{t}\right)$. The retailer monitors its own inventory position in order to place an order. The inventory position at period $t\left(I P_{t}\right)$ is the sum of existing inventory (i.e. $I_{t}$ ), ordered quantities in all outstanding orders $\left(O O_{i j}\right)$ from all suppliers $i$ at period $t$, and backorder at period $t$. These can be summarized mathematically as follows:

$$
\begin{aligned}
& I_{t}-I_{t-1} \cdot \sum_{i} I O_{t i}-d_{t} \\
& I P_{t}-I_{t} \cdot \sum_{i} O O_{t i}+B O t
\end{aligned}
$$

- Reorder cycle policy is employed to decide an order should be placed or not. Review period $T$ can be chosen using procedures analogous to those used for determining Economic Order Quantity (EOQ) such that $T$ is given by:

$$
T=\sqrt{\frac{z k}{h \mu}}
$$

where $k, h$ and $\mu$ are ordering cost, inventory holding cost and mean of demand of the retailer respectively. At the review period, the retailer will place a job announcement 
with quantity requirement so that the suppliers will bid for the order. The quantity $Q$ is calculated by equation (4):

$$
Q=S-I P_{t}
$$

$S$ in equation (4) is known as the order-up-to-level. In stochastic model, the retailer needs to know the mean $(\mu)$ and standard deviation $(\sigma)$ of its demand function, or the retailer can forecast them from past data, in order to calculate $S$. Forecasting error will be introduced if the retailer does not know its parameters well. Since the focus of this study is to investigate the effect of uncertainties, an assumption is made here that the retailer knows its parameters of the demand function well. In this connection, forecasting error is not introduced and it will not affect the comparison between the stochastic model and the proposed coordination mechanism later in Section 4. In this connection, the order-up-to-level is calculated as follows:

$$
S=\mu(T+L)+v \sigma \sqrt{(T+L)}
$$

where $v$ is the service level that the retailer would like to achieve and $L$ is the order lead time, which is assumed to be deterministic by the retailer (the retailer and suppliers are distributed in the sense that the retailer does not know the capacity function of the suppliers). The latter term in equation (5) is known as safety stock, which is a buffer to deal with demand uncertainty. The higher the service level, the higher is the safety stock. Assuming backorder cost of the retailer is $b$, and inventory holding cost is $h$ as defined above, the service level can be calculated by:

$$
v=\Phi^{-1}\left[\frac{b}{b+h}\right]
$$

where $\Phi($.$) is the probability density function of normal$ distribution. If there is no uncertainty exists in the system, above analysis is in fact equivalent to the analysis of EOQ.

- The suppliers will base on their own capacity and outstanding order to come up with the proposed bids. Essentially, all suppliers can submit a bid but the lead time is different for different suppliers. Without loss of generality, transportation lead time is incorporated in the production lead time so that the former is assumed to be zero. This is a common assumption in the literature. Assume the longest due date of supplier $i$ at period $t$ is $D_{i t}$ in its outstanding order, the expected delivery due date can be calculated by equation (7):

$$
\text { Expected Delivery Due Date }=D_{i t}+\frac{Q}{\text { Mean Capacity }}
$$

In addition, supplier inventory in increased by its daily capacity, which is also modeled as normal distribution.

- After the retailer received all the bids, the one with the shortest delivery due date will be selected.

- In the meantime, suppliers will update their inventories according to their daily capacity if there is any outstanding order.

- In addition, the retailer will check its outstanding orders in each period to identify if due date of a particular order reaches. In such a case, the retailer will ask the supplier to ship the order. In case the supplier does not have enough inventories to satisfy the order, penalty cost per unit (this is analogous to the backorder cost in case the agent is a retailer) will be charged to the supplier.

- At the end of each period, the inventory cost, backorder cost, and penalty cost of each agent are updated. At the end of the simulation, the operating cost of each agent, and hence the total system cost, can be recorded. In addition, setup cost per order is charged to the retailer and the supplier who gets the offer.

\subsection{Coordinated model}

From equation (4), the order quantity in stochastic model in fact consists of a basic quantity plus a safety stock. The rationale behind is to use the safety stock as a buffer to compensate the effect of uncertainties. Therefore, the stochastic model inherently increases inventory cost. In other words, the stochastic model is not dynamic enough because demand may be lower due to its random nature. In this connection, quantity flexibility is introduced in the coordinated model in order to provide the flexibility to the retailer, as well as suppliers to react with system dynamics. In order to apply this proposed mechanism, the supply chain members must be coordination oriented, but no explicit information sharing is required. In the coordinated model, similar procedures are followed as in the stochastic model, but with the following alteration:

- When a job is announced, it consists of a range of quantity required instead of a fixed quantity. Equation (5) can be rewritten as the following equations:

$$
\begin{gathered}
S=\mu(T+L)+\nu \sigma \sqrt{(T+L)}=A+B \\
A=\|(T+L) \\
B=v \sigma \sqrt{(T+L)}
\end{gathered} .
$$

The range of quantity $Q$ is defined such that: 


$$
Q \in[A-B, A+B]
$$

Equation (11) defines the "domain" of the variable "quantity" that the retailer requires the supplier to be shipped. In addition, the retailer will calculate a range of delivery dates so that supplier should ship the quantity as defined in equation (11) within the range of deliver due dates. The range of delivery date can be defined as in equation (12):

[Expected Delivery Due Date - $(B / \mu)$, Expected Delivery Due Date $+(B / \mu)]$

where Expected Delivery Due Date is given by equation (7)

- The procedure is the same as the stochastic model until lower bound of the due date in equation (12) of an outstanding order reaches. The retailer starts to coordinate with the supplier when and how many to be shipped as outline in Figure 1.

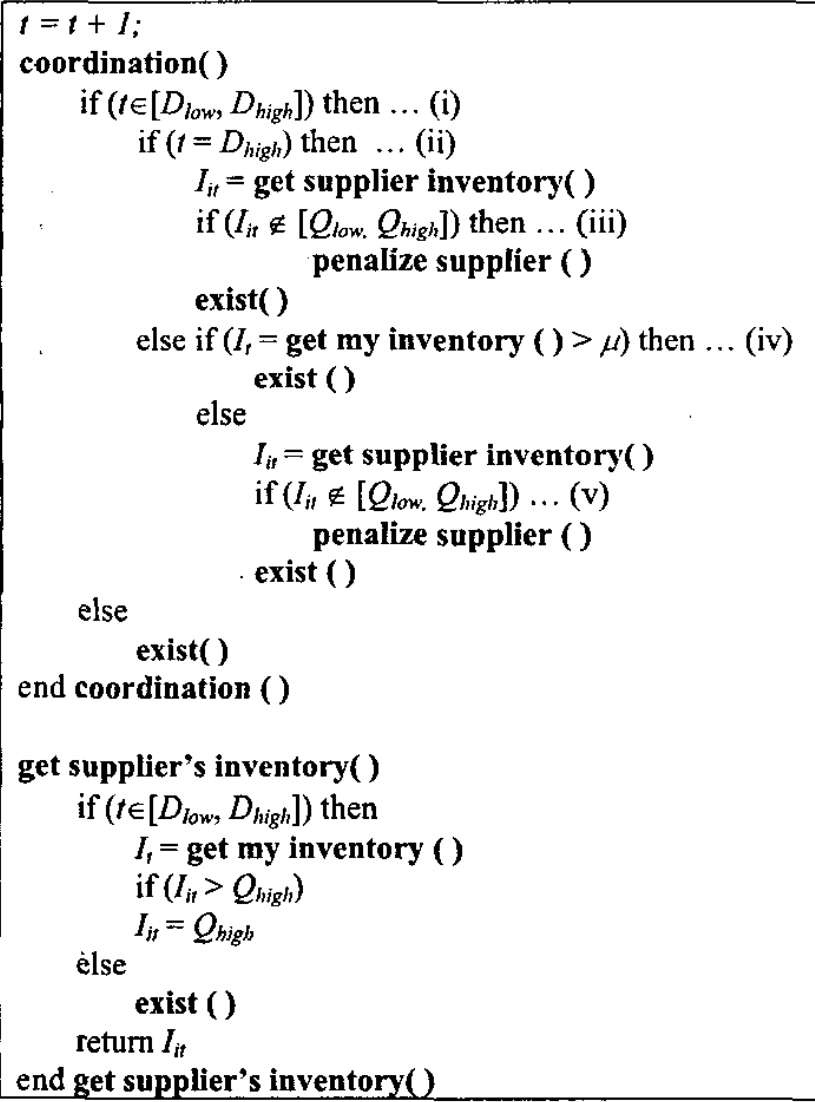

Figure 1. An outline of pseudo code for coordination

This can be formulated as a DCSP with two variables - one is quantity $Q$, and the other is the date for shipment $D$. The two variables are distributed among the retailer and supplier under contract. Domain of $Q$ is given by equation (11) and let $Q_{\text {low }}$ (i.e. A - B in equation (11)) and $Q_{\text {high }}$ (i.e. $A+B$ in equation (11)) be the lower bound and upper bound respectively. Domain of the date for shipment is given by equation (12) and let $D_{\text {low }}$ and $D_{\text {high }}$ be the lower bound and the upper bound respectively. The objective is to solve this DCSP through coordination. Condition (i) in Figure 1 constrains the coordination to be taken place only if the due date is within the domain in equation (12). Condition (ii) ensures the coordination phase is ended when the upper bound of the due date in equation (12) reaches. In such case, outstanding order must be completed. Condition (iv) makes sure the retailer does have enough inventory if no shipment is made when $D_{h i g h}$ is not reached. Please be reminded that conditions (iii) and (v) of the pseudo code allow the supplier to supply with quantity less than the defined domain, subjected to penalty being incurred, if the inventory of the supplier less than the lower bound as stated in equation (11). This is a constraint relaxation and hence the new domain of $Q$ is effectively become $\left(0, Q_{l o w}\right]$, i.e. any positive integer below $Q_{l o w}$. The reason to accept this argument is to ensure that the mechanism is complete and sound, i.e. the algorithm can always returns a solution. Of course, both the retailer and the suppliers would not like to relax the constraint, if possible, because both will suffer - the retailer gets less product and the suppliers make a loss due to the penalty.

\section{Results and discussions}

Simulation program is written in JAVA. The length of the simulation program is 465 periods while the first 100 periods is disregard to eliminate the error of the warm-up period. For each simulation setting, 10 runs, each with different random seed, were performed and the average of the 10 simulation runs was computed in order to minimize random errors.

Simulation results are depicted in Figures 2 to 3 . Each figure consists of three graphs: (a) the results of an idealistic system that does not take uncertainties into consideration; (b) the results obtained from the system employing stochastic model to deal with uncertainties; and (c) the results by employing the proposed coordination mechanism to react with uncertainties. Obviously, the performance of (a) is the worst subjected to uncertainties. However, this is included solely as a benchmark so that improvement of the stochastic model and the proposed mechanism from this worst case can be compared. All grouped graphs under the same heading are drawn with the same scale so that they can be compared visually in an easy manner. The two base axes that are labeled with "demand uncertainty" and "capacity uncertainty" refer to the two independent variables, retailer's demand and suppliers' capacity respectively, in the simulation study. They are varied by altering the standard deviation of the associated normal distribution to reflect the level of uncertainties of 
each variable. The higher the value of the parameters, the larger is the standard deviation of the normal distribution, i.e. more uncertain. On top of the findings on indivicual measurement that will be discussed, it is found that the performance under certain environment, i.e. no uncertainties present, is the best as expected. Therefore, all simulation results are presented relative to this situation, but the word "relative" will not be stated explicitly in most of the case.

\subsection{Total system cost}

Figure 2a shows that the total system cost is increased in the same direction as both demand and capacity uncertainties. In fact, this observation is trivial as level of uncertainty is increasing, the chance that the system is out of stock is also increasing, which leads to increase in backorder cost.

If stochastic model is employed, safety stock is used as buffer to reduce the impacts of the uncertainties so that it is easily to conclude from Figure $2 b$ that the total system cost is improved subjected to demand and capacity uncertainties. Improvement is more significant in highly uncertain environment. Although the total system cost is reduced, the trend that total system cost increase with level of uncertainty is still valid. In other words, the safety stcck is a means to reduce loss due to uncertainties, but it is not so effective to eliminate system dynamics completely.

If the proposed coordination mechanism is employed, the total system cost is even improved to a larger extent than the system with stochastic model as shown in Figure 2c. This is probably due to the dynamic nature of the proposed algorithm that can successfully filter the system dynamics. In addition, the proposed algorithm is not only able to reduce the total system cost but the impact of increasing uncertainty is also suppressed. That means the marginal cost against uncertainty (i.e. the cost increased "yy increasing one level of uncertainty) is decreased significantly. This can be shown by comparing the deviation in the height of various columns of the graphs in Figure 2.

\subsection{Fill rate}

The second performance measure to be examined is fill rate, which is usually not considered in mathematical programming. Figure 3 illustrates the performance of the three systems in terms of fill rate. In order to ease readers to study the graphs, the two axes of uncertainty are rearranged. From Figure $3 a$, it can be observed that fill rate (the higher is the better) is deteriorating while uncertainties are increasing. This is not surprising because the higher the uncertainty, the easier is the system encounters stock out, i.e. customer orders could not be filled. In the stochastic model (Figure 3b), since safety stock is employed as cushion to uncertainties, fill rate is improved as compared with Figure 3a. However, the improvement is less than 3\% in fill rate.

On the other hand, for the coordinated system, the fill rate can be improved more than $5 \%$ as compared with the idealistic system. In addition, the maximum benefit that switches from stochastic model to the proposed model is as high as $3.5 \%$ improvement in fill rate. If the average improvement among all uncertainties setting is considered, it is found that the average improvement of stochastic model over the idealistic model is $1.2 \%$ against $3.6 \%$ of the proposed coordination algorithm.

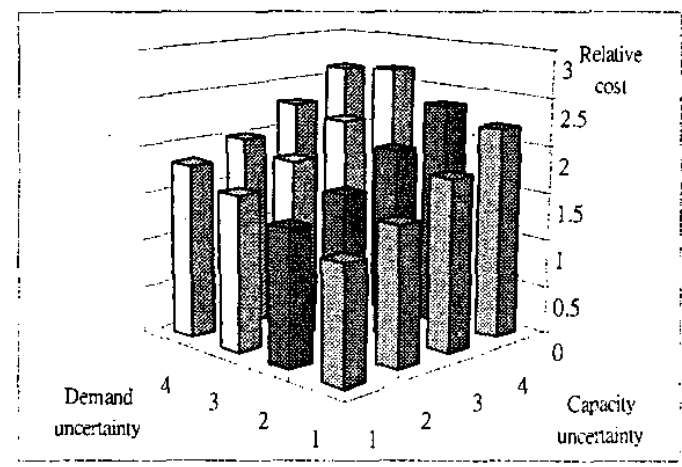

(a) Idealistic system

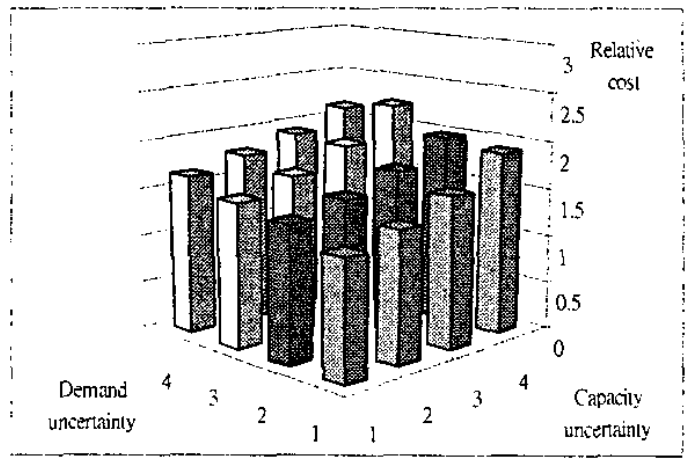

(b) Stochastic system

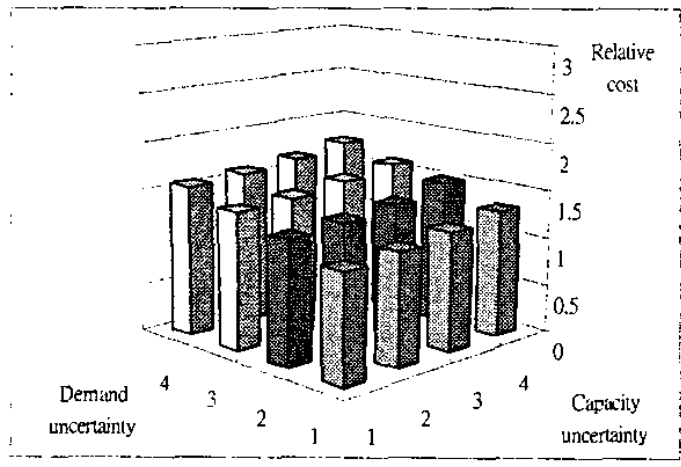

(c) Coordination system

Figure 2. Relative total system cost against uncertainties 


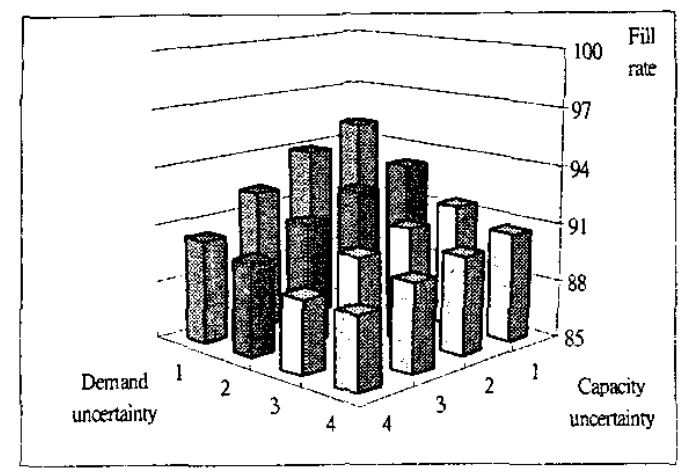

(a) Idealistic system

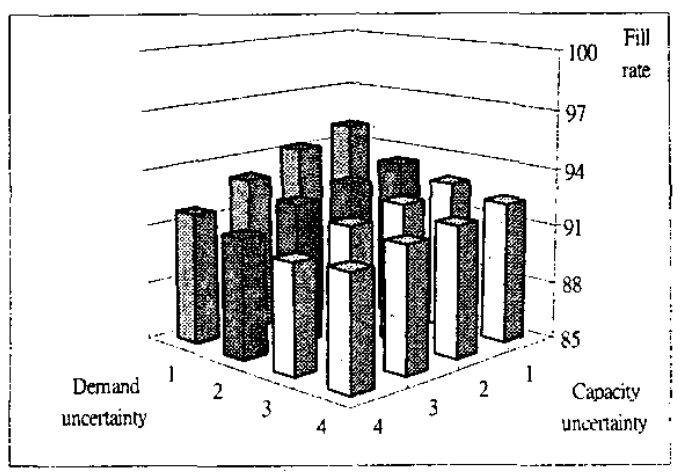

(b) System with stochastic modeling

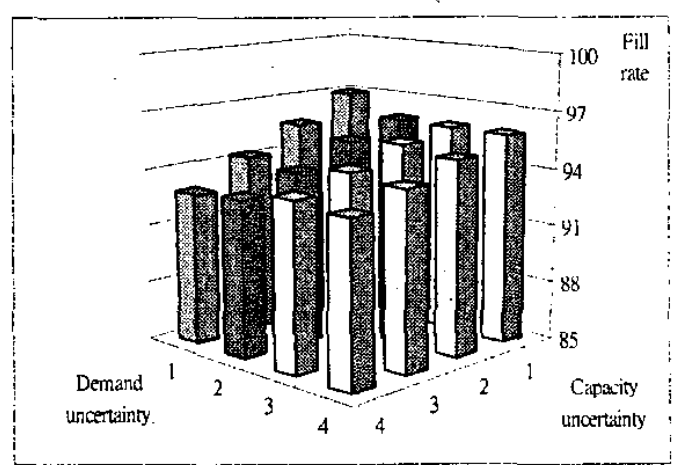

(c) System with the proposed coordination mechanism

Figure 3. Fill rate against uncertainties

\section{Conclusions}

'System dynamics in supply chains is not uncommon. Facing dynamics situation in modern distributed supply chains, traditional stochastic model may not be dynamic enough to react with uncertainties from different participating members in the supply chains. In this paper, an innovative coordination mechanism is proposed for distributed supply chains for inventory management so that delivery decision of outstanding order is negotiable. This dynamic nature is proven, through simulation study, to be effectively in reducing total supply chain cost and improving fill rate. More effort can be paid to enhance the robustness of the proposed mechanism.

\section{References}

[1] J. Gjerdrum, N. Shah, and L. G. Papageorgiou, "A combined optimisation and agent-based approach to supply chain modelling and performance assessment", Production Planning and Control, Vol 12, No. 1, pp. 81-88, 2001.

[2] M. Tambe, J. Adibi, Y. Al-Onaizan, A. Erdem, G. A. Kaminka, S. C. Marsella, and I. Muslea, "Building agent teams using an explicit teamwork model and learning", Artificial Intelligence, Vol 110, No. 2, pp. 215-239, 1999.

[3] S. Viswanathan, and R. Piplani, "Coordinating supply chain inventories through common replenishment epochs", European Journal of Operational Research, Vol 129, No. 2, pp. 277-286, 2001.

[4] J. Chen, and L. Xu, "Coordination of the Supply Chain of Seasonal Products", IEEE Transactions on Systems Man, and Cybernetics - Part A, Vol 31, No.6, pp. 524-531, 2000.

[5] A. A. Tsay, "The Quantity Flexibility Contract and Supplier-Customer Incentives", Management Sciences, Vol 45, No. 10, pp. 1339-1358, 1999.

[6] J. M. Swaminathan, S. F. Smith, and N. M. Sadeh, "Modeling Supply Chain Dynamics: A Multiagent Approach", Decision Sciences, Vol 29, No. 3, pp. 607-632, 1998.

[7] N. M. Sadeh, D. W. Hildum, D. Kjenstad, and A. Tseng, "MASCOT: an agent-based architecture for dynamic supply chain creation and coordination in the internet economy", Production Planning \& Control, Vol 12, No. 3, pp. 212-223, 2001.

[8] M. Yokoo, E. H. Durfee, T. Ishida, and K. Kuwabara, "The Distributed Constraint Satisfaction Problems: Formalization and Algorithms", IEEE Transactions on Knowledge and Data Engineering, Vol 10, No. 5, pp. 673$685,1998$.

[9] M. Yokoo, T. Ishida, E. H. Durfee, and K. Kuwabara, "Distributed Constraint Satisfaction for Formalizing Distributed Problem Solving", Proceedings of IEEE International Conference on Distributed Computer Systems, pp. 614-621, 1992.

[10] F. T. S. Chan, and H. K. Chan, "A New Model for Manufacturing Supply Chain Networks: Multi-Agent Approach", Proceedings of the Institution of Mechanical Engineers Part B: Journal of Engineering Manufacture, Vol 218, No. 4, pp. 443-454, 2004. 